\title{
Long-term functional results of first metatarsophalangeal joint arthrodesis for hallux rigidus
}

\author{
D.V. Ilchenko ${ }^{1}$, A.V. Korolev ${ }^{1,2}$, A.A. Kardanov ${ }^{1,2}$ \\ ${ }^{1}$ European Clinic of Sports Traumatology and Orthopaedics (ECSTO), Moscow, Russian Federation, \\ ${ }^{2}$ Peoples' Friendship University of Russia, Moscow, Russian Federation
}

\begin{abstract}
Introduction First metatarsophalangeal joint (MTPJ) osteoarthritis hallux rigidus (HR) is the most common arthritic condition in the foot and occurs in 2.5-7.8\% of the population over 50 years. First MTPJ arthrodesis is currently considered to be the gold standard for the treatment of end-stage HR. When treating the advanced stage of HR, arthrodesis has long been established as an effective surgical procedure providing very predictable and satisfying results. Although the surgical authors prefer joint-sparing procedures first MTPJ fusion when required is the joint destructive procedure of choice in severe HR to eliminate or minimize pain, stabilize the first MTPJ and allow the hallux to bear weight. Functional outcomes achieved with fusion are also consistent with those from other surgical procedures. The purpose of this study is to evaluate long-term functional outcomes of first MTPJ arthrodesis. Material and methods Clinical and functional outcomes of MTPJ arthrodesis performed for 19 patients (25 feet) with grade 3 or 4 HR using the Coughlin and Shurnas Classification System between September 2010 and December 2017 were reviewed. The patients' mean age was 60 years (range, 35 to 86 years). The median interval between surgery and the last follow-up was 5 years (interquartile range, 1.5 to 8 years). Ultimately, outcome assessment relied on patient satisfaction, radiographic examination, visual analogue scale (VAS), the American Orthopaedic Foot \& Ankle Society (AOFAS) and the Foot and Ankle Ability Measure (FAAM) scores. Results AOFAS score was rated as good in $92 \%$ of the cases $(23 / 25)$, as fair in $8 \%(2 / 25)$ with neither excellent nor poor results recorded. The median AOFAS score increased from preoperative 43.0 to postoperative 85.0 that was statistically significant $(\mathrm{p}<0.05)$. Postoperative median FAAM Activities of Daily Living (ADL) was $99 \%$, median subjective ADL was $90 \%$, median FAAM sport was $84 \%$, and median subjective sport was $80 \%$. Patient subjective assessment was scored as "excellent" in $36 \%$ of cases (9/25), "good" in $56 \%(14 / 25)$, "fair" in $8 \%(2 / 25)$ with no "poor" results recorded. The median VAS pain score decreased from 6 at baseline to 1 point postsurgery $(\mathrm{p}<0.05)$. Conclusion First MTPJ arthrodesis was shown to be a very effective, reliable and lasting primary procedure for severe HR that provided $92 \%$ of good outcomes in our series. The procedure can be advocated as a predictable and excellent option for Coughlin and Shurnas grades 3 and 4 HR facilitating high success rates in pain relief and restoration of function.
\end{abstract}

Keywords: hallux rigidus, first metatarsophalangeal joint osteoarthritis, osteotomy, arthrodesis

\section{INTRODUCTION}

Deforming osteoarthritis (OA) is a degenerative long-lasting joint disease affecting articular cartilage and periarticular tissues with alternating acute episodes of exacerbation and periods of remission. Deforming OA is the most common joint disorder. First metatarsophalangeal joint (MTPJ) osteoarthritis hallux rigidus is the most common arthritic condition in the foot. Of all patients aged over 50 years, 2.5$7.8 \%$ report degenerative arthritis of the first MTPJ, termed 'hallux rigidus' (HR) [1, 2]. It is characterised by pain, a progressive decrease in range of motion, primarily with dorsiflexion, periarticular osteophyte formation and cartilage degeneration. The disease initially affects the dorsal portion of the joint and progresses resulting in articular cartilage loss, debilitating pain and limited functional activity [3]. Although HR was first described in the orthopaedic literature towards the end of the nineteenth century, a search for more effective treatment options is still being conducted [4].

Nonsurgical techniques can often be used to successfully treat patients at early stages of degeneration whereas surgical treatment is associated with greater risk of complications or lower rate of satisfactory outcomes. Cheilectomy and arthrodesis of the first MTPJ remain first-line surgical treatments tested in clinical trials [5, 6, 7]. Premeditated ankylosis of the first MTP joint was described by Wyeth (1887) and later by Clutton (1894) who contended that ankylosis in an ideal position would produce a permanent and very satisfactory result. Glissan and McKeever are generally associated with the modern era of first MTP joint arthrodesis and they described unintentional ankylosis in a patient who 
suffered stiffness after bunion surgery and found that the 'complication' made the foot function better. It is from these observations that led to the orthopedic community to embrace first MTPJ arthrodesis in the mid-20th century. There are multiple surgical techniques for arthrodesis of the first MTPJ utilizing different ways to prepare the bone surfaces and a wide variety of techniques of internal and external fixation, all of which may have their place in various instances [5]. Dorsal plate and lag screw fixation provides reliable biomechanical stability for the fusion $[8,9,10]$. The reported rate of bone healing and consolidation of the fusion site ranges between $77 \%$ and $100 \%$ with the mean of $90 \%[11,12,13]$. Optimal position of the first MTP joint is with neutral rotation $\mathrm{R} 1,5^{\circ}$ to $15^{\circ}$ of valgus and $20^{\circ}$ to $25^{\circ}$ relative to the first metatarsal M1 [11, 14]. Different types of arthroplasty and arthrodesis of the first MTPJ can be the method of choice in degenerative joint surgery. Many replacement procedures are associated with a higher complication rate due to bone loss, loosening or fragmentation of implant components and transfer metatarsalgia [15]. Traditionally, first MTPJ arthrodesis can be applied to primary degenerative arthritis and revision of prior failed surgery to this joint with iatrogenic changes [16, 17]. However, revisionary surgery of the first MTP joint has been shown to result in inferior clinical and functional outcomes and greater complications [15]. Primary arthrodesis of the first MTP appears to be a safe and efficient method for surgiucal treatment of severe arthritis. The procedure can facilitate bone healing that removes pain, regains range of movement, maintains the medial column and toe length, achieves stability of the first ray sacrificing motion in the MTPI. Furthermore, fusion sacrifices motion which may lead to patient dissatisfaction and a decrease in first-ray mobility results in a significant change in the postoperative gait pattern, characterized by a shorter step length and a reduction in ankle power at pushoff. Clinical results indicate effective pain relief and a high activity level in patients with symptomatic HR.

The purpose of this study was to evaluate longterm functional outcomes of first MTPJ arthrodesis.

\section{MATERIAL AND METHODS}

We performed a retrospective study of 19 patients (25 feet) who had undergone a surgical treatment for deforming OA of the first MTP joint between September 2010 and December 2017 at the European Clinic of Sports Traumatology and Orthopaedics of the European Medical Center. The mean patients' age was 60 years (range, 35 to 86 years). There were 1 male and 18 female patients. Preoperative radiological examination included the weightbearing dorsiplantar and lateral foot films. HR grage $4(\mathrm{n}=14)$ and HR grade $3(n=11)$ using the Coughlin and Shurnas Classification System were diagnosed using clinical and radiological findings [11] (Fig. 1).

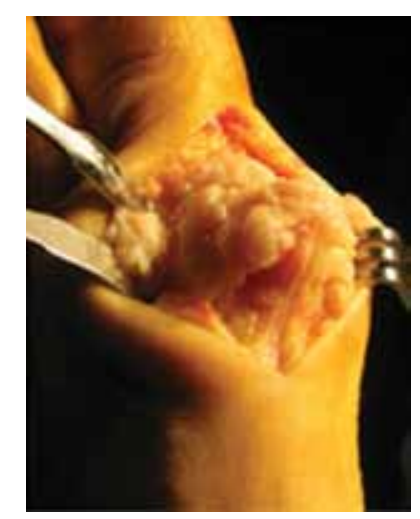

Fig. 1 Severe deforming OA of the first MTP joint Coughlin и Shurnas grade 4
Preoperative planning included measurements of the metatarsal index and interphalangeal valgus and evaluation of degenerative changes on weightbearing radiographs. Isolated arthrodesis of the first MTPJ was performed for 18 patients, and the Chevron osteotomy was added in one case with M1R $1 \leqslant 30^{\circ}$ and $\mathrm{M} 1 \mathrm{M} 2 \leqslant 12^{\circ}$ (Fig. 2), and the distal minimally invasive osteotomy R1 supplemented 6 operative interventions to correct hallux valgus interphalangeus with the bone fixed with screws.

All patients were examined preoperatively and at a long-term follow-up. The median interval between surgery and the last follow-up was 5 years (interquartile range, 1.5 to 8 years). Patients were requested to complete the American Orthopaedic Foot \& Ankle Society (AOFAS) questionnaire preoperatively and at a long-term follow-up to assess the forefoot (Table 1), and the Foot and Ankle Ability Measure (FAAM) to assess postoperative physical functioning [18].

Outcome assessment also relied on subjective patient satisfaction after surgical treatment rated as 'excellent', 'good', 'fair' and 'poor' and preoperative and postoperative visual analogue scale (VAS) score. 

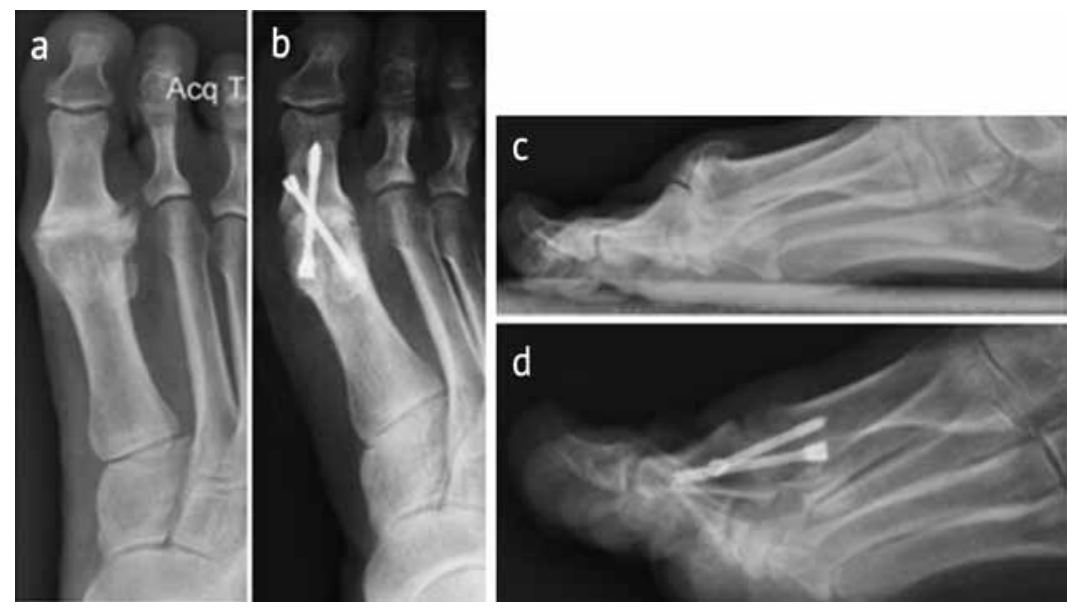

Fig. 2 Preoperative $(\boldsymbol{a}, \boldsymbol{c})$ and postoperative $(\boldsymbol{b}, \boldsymbol{d})$ radiographs of the right foot showing the foot bones before and after first MTPJ arthrodesis combined with the Chevron osteotomy of M1
Table 1

Interpretation of AOFAS scores

\begin{tabular}{|c|c|}
\hline Rating & Scores \\
\hline Excellent & $95-100$ \\
\hline Good & $75-94$ \\
\hline Fair & $51-74$ \\
\hline Poor & $0-50$ \\
\hline
\end{tabular}

\section{Statistical analysis}

Statistical analysis was performed with computer software (IBM SPSS Statistics 23.0.0.0). The Shapiro-Wilk test was used to determine normality of distribution in the groups. The data obtained were summarized as means \pm standard deviations with maximal and minimal values in normal distribution; numerical data were presented with median and interquartile range when distribution was different from the norm. Quantitative data were presented in box plots. The Wilcoxon signed-rank test was used to analyze data in two related paired samples. The Mann-Whitney $\mathrm{U}$ test was used to compare differences between two independent groups. For calculations, a significance level of $5 \%(p \leqslant 0.05)$ was adopted.

\section{Surgical technique}

Different types of fixation and techniques for preparation of the articular surfaces are employed for first MTPJ arthrodesis. We applied two different techniques depending on a ratio between M1 and M2 lengths. The start-up surgical phase was identical for the cases with medial approach followed by dissection of the articular capsule. R1 and M1 articular ends were resected with M1 length being greater or equal to M2 length to regain metatarsal index and fixation provided either with two crossed compression screws or a dorsal plate and a lag screw. The latter was shown to be a more stable and more expensive option.

Fixation was ensured with screws only $(n=21)$ and a plate and a screw $(n=4)$. Position of metal constructs was evaluated radiologically in all cases (Fig. 3). Milling of the articular ends was produced with M1 length not exceeding M2 length thoroughly observing volume of resection to avoid excessive shortening of M1 and development of transfer metatarsalgia. Excised bone fragments were removed at the stage and conjugated ends adapted to ensure a tight M1 and R1 contact. Compression screws were used for bone fixation. Optimal positioning of R1 relative to M1 is very important. A supporting foor plate was used to ensure ankle dorsiflexion at $90^{\circ}$ and neutral rotation $\mathrm{R} 1,5^{\circ}$ to $15^{\circ}$ of valgus and $20^{\circ}$ to $25^{\circ}$ relative to the first metatarsal M1 (Fig. 4). In our series, successful arthrodesis occurred in all cases after operative treatment of HR and bone healing was radiologically visualized at 3 to 4 months postsurgery.

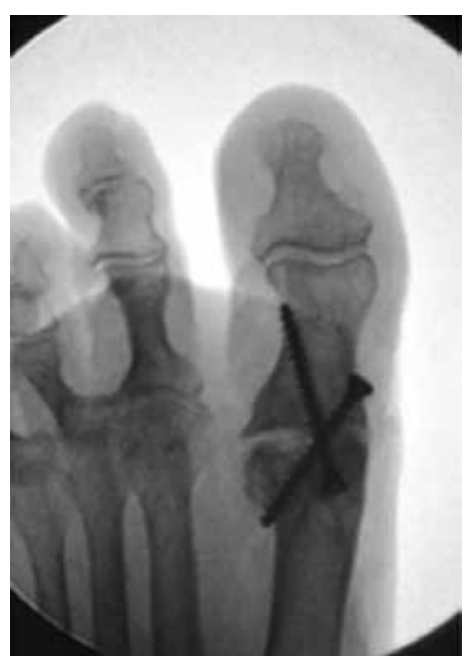

Fig. 3 Position of metal construct identified with intraoperative radiograph

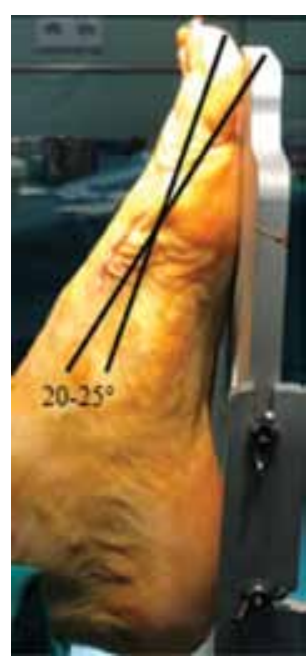

Fig. 4 Supporting foot plate used to secure intraoperative position of the foot. A R1 to M1 angle is supposed to measure $20-25^{\circ}$ 


\section{RESULTS}

AOFAS scores showed $92 \%$ of good results (23 operative interventions) and $8 \%$ of fair (2 surgeries) results. Neither excellent nor poor outcomes were observed. Absence of excellent results to be rated on AOFAS scale was associated with considerable limitations in ROM in the first MTPJ because of fusion with the resultant score lacking 10 points and being rated as a good result. The median AOFAS score was 43.0 (interquartile range, from 25.0 to 47.0 ) that increased postsurgery to 85.0 (interquartile range, from 82.0 to 87.0 ) and was statistically significant $(\mathrm{p}<0.05)$. Preoperative and postoperative AOFAS scores are presented in Figure 5. Subjective patient satisfaction was rated as 'excellent' in $36 \%$ of the cases ( 9 surgical interventions), good' in $56 \%$ (14 surgical interventions) and 'fair' in $8 \%$

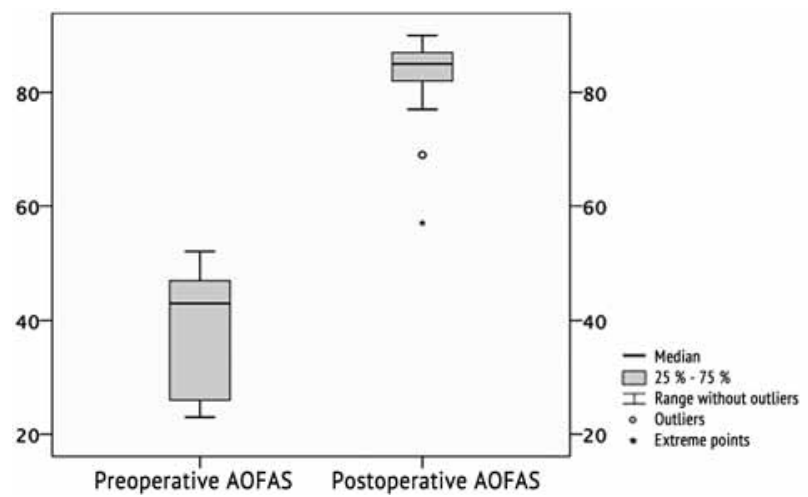

Fig. 5 Preoperative and postoperative AOFAS scores

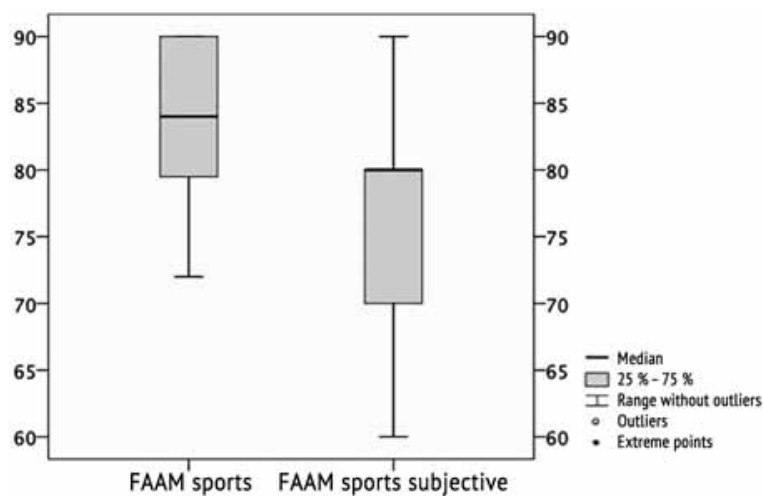

Fig. 7 FAAM scores on sports subscale
(2 surgical interventions). No 'poor' subjective scores were recorded. The median postoperative FAAM score on activities of daily living subscale was 99 \% (interquartile range, from 98 to $100 \%$ ) and on subjective form of activities of daily living subscale was $90 \%$ (interquartile range, from 80 to $100 \%$ ) (Fig. 6). The FAAM sports subscale was scored only in 7 cases of first MTPJ arthrodesis. The median postoperative FAAM score on sports subscale was $84 \%$ (interquartile range, from 78 to $90 \%$ ) and on subjective sports form was $80 \%$ (interquartile range, from 70 to $80 \%$ ) (Fig. 7). The median VAS score was 6 (interquartile range, from 4 to 7 ) preoperatively and improved to 1 point postoperatively (interquartile range, from 1 to 1$)$ that was statistically significant $(p<0.05)$ (Fig. 8).

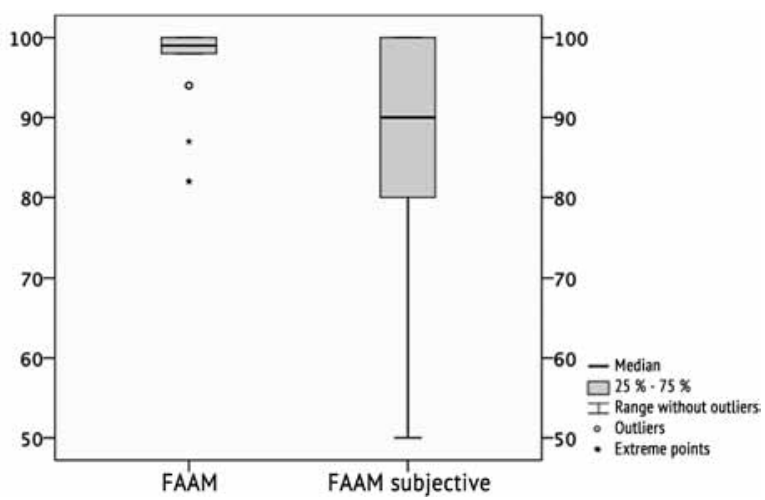

Fig. 6 FAAM scores on ADL subscale

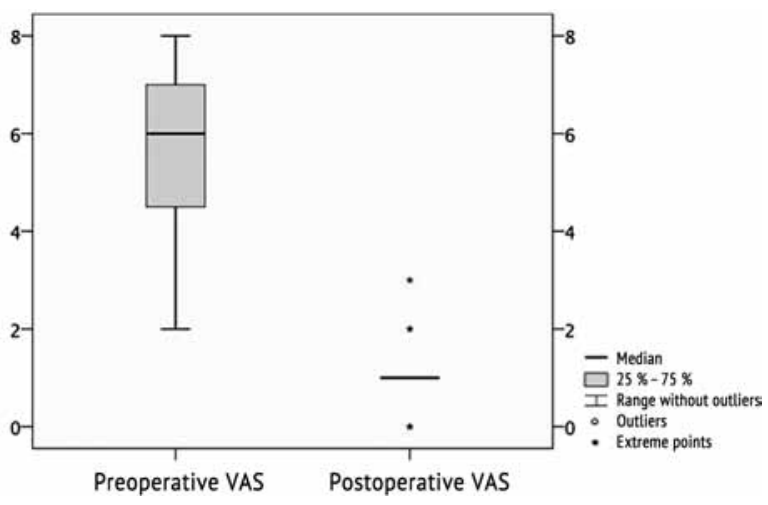

Fig. 8 Preoperative and postoperative VAS scores

\section{DISCUSSION}

SevereOAof the firstMTPJ remains difficulttotreat. First MTPJ arthrodesis is a well-documented, reliable and reproducible procedure that results in excellent pain relief and improved functionality, however, loss of motion can lead to limited activities of daily living or sports. However, patients may experience shoe- wearing limitation after the procedure. There are still debates regarding arthrodesis and arthroplasty aimed at preservation of motion in the first MTPJ and, subsequently, biomechanics of the gait. Implant arthroplasty of the first MTPJ has a high complication rate due to greater loss of osseous tissues, loosening 
and tear and wear of prosthetic components as well as absence of outcome prediction and limited number of clinical trials.

In our series, first MTPJ arthrodesis has been shown to be a very effective and reliable procedure for HR providing excellent clinical and functional results. Our findings were comparable to those reported in foreign studies. Raikin et al. reported $82 \%$ good results and $4 \%$ poor outcomes in 27 cases of first MTPJ arthrodesis at a 30-month follow-up with VAS score decreased to 0.7 points and AOFAS score increased to 83.8 points [19]. Gibson and Thomson reported $95.5 \%$ good results in 22 patients (38 feet) following first MTPJ arthrodesis at a 24-month follow-up with VAS score decreased to 1.1 points and all arthrodeses united [20]. Stevens et al. presented systematic review of the literature including thirtythree studies, describing a total of 741 arthrodeses and 555 total joint replacements. Six different prostheses were used for total joint replacement, and various fixation techniques were used for arthrodesis. Pooled results showed superiority of arthrodesis compared with total joint replacement for improving clinical outcome (by 43.8 versus 37.7 points on the AOFAS-HMI score) and reducing pain (a decrease of 6.56 versus 4.65 points on the VAS pain score). Fewer intervention-related complications (23.1\% versus $26.3 \%$ ) and revisions (3.9\% versus $11 \%$ ) were reported after arthrodesis as compared with total joint replacement [21]. Coughlin and Shurnas reported $100 \%$ positive outcomes in 30 patients with Grade-3 HR and Grade-4 HR treated with first MTPJ arthrodesis, with AOFAS scores averaged 88.9 at an average follow-up of 80 months [11]. Chraim et al. performed a retrospective review of 60 patients (61 feet) who underwent arthrodesis of the first MTPJ with average follow-up of 47.3 months. The AOFAS increased postoperatively from 40.9 to 79.3. Concerning the postoperative satisfaction, $71.7 \%$ of the patients were very satisfied and $18.3 \%$ were satisfied. Complications included $6.7 \%$ of the cases who ended up with a painless pseudarthrosis and required no additional surgery [22]. Successful consolidation were reported in $98 \%$ of primary first MTPJ arthrodesis [23, 24, 25]. Wanivenhaus et al. restrospectively reviewed 39 patients (41 feet) who underwent first MTPJ fusion using a compressive locking plate that was successful in
$95 \%$ of the cases with the forefoot AOFAS score improved significantly from $50 \pm 13$ preoperatively to $80 \pm 7$ at $>1$ year of follow-up $(p=0.001)$ [26] Korim et al. performed a qualitative synthesis of the current literature to determine the union frequencies for first MTPJ arthrodesis as well as the influence of pathology, joint preparation and fixation methods on union. The systematic review included 26 studies with 2059 feet. The mean age was 60 years (range 18-84) and the mean follow-up was 32.6 months (range 1.5-156). The union frequency was $93.5 \%$ (1923/2059). The union frequencies were significantly higher when low velocity joint preparation methods were used $(p<0.0001)$ and the pathology was HR $(p=0.002)$ [27].

Our findings have been found to be in line with those reported in the literature. Patients with Coughlin and Shurnas Grade 3 and Grade 4 HR in our series showed lower VAS values, higher AOFAS scores and levels of activities of daily living and sports as well as higher patient satisfaction at a long-term followup. Brodsky et al. examined the effects of first MTP joint arthrodesis on activities of daily living and recreational activities in 53 patients (60 feet). The mean follow-up was 44 months. The authors found that patients were able to return to hiking $92 \%$ of the time, golf $80 \%$ of the time, tennis $75 \%$ of the time and jogging $75 \%$ of the time [28]. Similar results were obtained by Da Cunha et al. who assessed sports and physical activities in 50 patients following first MTPJ arthrodesis identified by review of a prospective registry with follow-up at a mean of 5.1 years with a mean age at surgery of 49.7 years. The most common sports and physical activities patients participated in were walking, biking, weightlifting, swimming, running, and golf. Compared to preoperatively, patients rated $27.4 \%$ of activities as less difficult, $51.2 \%$ as the same, and $21.4 \%$ as more difficult. Ninety-six percent of patients (48/50) were satisfied with the procedure regarding return to sports and physical activities [29]. In our series, we could evaluate sports and physical activities in 7 patients following surgical treatment of HR with the FAAM and patient satisfaction levels dropped down to $84 \%$ and $80 \%$, respectively.

Our study had several limitations. First, this was a retrospective case series with a small number of participants. Second, clinical signs of overloading 
or OA in adjacent joints of the first ray or joints of the foot might have played a role in postoperative AOFAS and FAAM scores in presence of limited mobility in the first MTPJ following arthrodesis. Third, a further thorough functional analysis is needed to be performed with findings from pedobarography and preoperative and postoperative gait analysis as far as objective AOFAS and FAAM scores have not been confirmed by trials and cannot be used for informative conclusions. We can also suggest that a more continuous observation of surgically treated patients would be practical for comprehensive assessment of clinical and radiological findings.

\section{CONCLUSION}

First MTPJ arthrodesis was shown to be be advocated as a predictable and excellent option a very effective, reliable and lasting primary for Coughlin and Shurnas grades 3 and $4 \mathrm{HR}$ procedure for severe HR that provided $92 \%$ of facilitating high success rates in pain relief and good outcomes in our series. The procedure can restoration of function.

Competing interests. The authors have declared that no competing interest exists.

\section{REFERENCES}

1. Razik A., Sott A.H. Cheilectomy for Hallux Rigidus. Foot Ankle Clin., 2016, vol. 21, no. 3, pp. 451457. DOI: 10.1016/j. fcl.2016.04.006.

2. Roddy E., Thomas M.J., Marshall M., Rathod T., Myers H., Menz H.B., Thomas E., Peat G. The population prevalence of symptomatic radiographic foot osteoarthritis in community-dwelling older adults: cross-sectional findings from the clinical assessment study of the foot. Ann. Rheum. Dis., 2015, vol. 74, no. 1, pp. 156-163. DOI: 10.1136/annrheumdis-2013-203804.

3. Meyer J.O., Nishon L.R., Weiss L., Docks G. Metatarsus primus elevatus and the etiology of hallux rigidus. J. Foot Surg., 1987, vol. 26, no. 3, pp. 237-241.

4. Therman H., Becher C., Kilger R. Hallux rigidus treatment with cheilecomy, extensive plantar release, and additional microfracture technique. Techn. Foot Ankle Surg., 2004, vol. 3, pp. 210-217. DOI: 10.1097/00132587-200412000-00004.

5. Kumar S., Pradhan R., Rosenfeld P.F. First metatarsophalangeal arthrodesis using a dorsal plate and a compression screw. Foot Ankle Int., 2010, vol. 31, no. 9, pp. 797-801. DOI: 10.3113/FAI.2010.0797.

6. Ho B., Baumhauer J. Hallux rigidus. EFORT Open Rev., 2017, vol. 2, no. 1, pp. 13-20. DOI: 10.1302/2058-5241.2.160031.

7. Berezhnoi S.Iu. Artroz pervogo pliusnefalangovogo sustava: chreskozhnoe operativnoe lechenie, vybor khirurgicheskoi metodiki, kliniko-rentgenologicheskaia klassifikatsiia [First metatarsophalangeal arthrodesis: percutaneous surgical treatment, choice of surgical technique, clinical-and-roentgenological classification]. Travmatologiia i Ortopediia Rossii, 2017, vol. 23, no. 1, pp. 8-22. (in Russian) DOI: 10.21823/2311-2905-2017-23-1-8-22.

8. Politi J., John H., Njus G., Bennett G.L., Kay D.B. First metatarsal-phalangeal joint arthrodesis: a biomechanical assessment of stability. Foot Ankle Int., 2003, vol. 24, no. 4, pp. 332-337. DOI: 10.1177/107110070302400405.

9. Hyer C.F., Glover J.P., Berlet G.C., Lee T.H. Cost comparison of crossed screws versus dorsal plate construct for first metatarsophalangeal joint arthrodesis J. Foot Ankle Surg., 2008, vol. 47, no. 1, pp. 13-18. DOI: 10.1053/j.jfas.2007.08.016.

10.Cone B., Staggers J.R., Naranje S., Hudson P., Ingram J., Shah A. First Metatarsophalangeal Joint Arthrodesis: Does the Addition of a Lag Screw to a Dorsal Locking Plate Influence Union Rate and/or Final Alignment after Fusion. J. Foot Ankle Surg., 2018, vol. 57, no. 2, pp. 259-263. DOI: 10.1053/j.jfas.2017.09.003.

11.Coughlin M.J., Shurnas P.S. Hallux rigidus. Grading and long-term results of operative treatment. J. Bone Joint Surg. Am., 2003, vol. 85, no. 11, pp. 2072-2088.

12.Marks R.M. Arthrodesis of the first metatarsophalangeal joint. Instr. Course Lect., 2005, vol. 54, pp. 263-268.

13.Bennett G.L., Kay D.B., Sabatta J. First metatarsophalangeal joint arthrodesis: an evaluation of hardware failure. Foot Ankle Int., 2005, vol. 26, no. 8, pp. 593-596. DOI: 10.1177/107110070502600803.

14.Mazalov A.V., Zagorodnii N.V., Protsko V.G., Sultanov E.M., Khamokov Z.Kh. Khirurgicheskoe lechenie tiazhelogo (2-3 stepeni) deformiruiushchego artroza pervogo pliusnefalangovogo sustava: zadachi, podkhody, tekhnika [Surgical treatment of severe (Degree 2-3) deforming arthrosis of first metatarsophalangeal joint: tasks, approaches, technique]. Travmatologiia i Ortopediia Rossii, 2011, no. 4 (62), pp. 69-76. (in Russian) Available at: https://doi.org/10.21823/2311-2905-2011--4-69-76.

15.Gross C.E., Hsu A.R., Lin J., Holmes G.B., Lee S. Revision MTP arthrodesis for failed MTP arthroplasty. Foot Ankle Spec., 2013, vol. 6, no. 6, pp. 471-478. DOI: 10.1177/1938640013502725.

16.Lombardi C.M., Silhanek A.D., Connolly F.G., Dennis L.N., Keslonsky A.J. First metatarsophalangeal arthrodesis for treatment of hallux rigidus: a retrospective study. J. Foot Ankle Surg., 2001, vol. 40, no. 3, pp. 137-143.

17.Womack J.W., Ishikawa S.N. First metatarsophalangeal arthrodesis. Foot Ankle Clin., 2009, vol. 14, no. 1, pp. 43-50. DOI: 10.1016/j. fcl.2008.11.008.

18.Martin R.L., Irrgang J.J., Burdett R.G., Conti S.F., Van Swearingen J.M. Evidence of validity for the Foot and Ankle Ability Measure (FAAM). Foot Ankle Int., 2005, vol. 26, no. 11, pp. 968-983. DOI: 10.1177/107110070502601113.

19.Raikin S.M., Ahmad J. Comparison of arthrodesis and metallic hemiarthroplasty of the hallux metatarsophalangeal joint. Surgical technique. J. Bone Joint Surg. Am., 2008, vol. 90, no. Suppl. 2, pt. 2, pp. 171-180. DOI: 10.2106/JBJS.H.00368.

20.Gibson J.N., Thomson C.E. Arthrodesis or total replacement arthroplasty for hallux rigidus: a randomized controlled trial. Foot Ankle Int., 2005, vol. 26, no. 9, pp. 680-690. DOI: 10.1016/s1619-9987(08)60258-5. 
21.Stevens J., De Bot R.T.A.L., Hermus J.P.S., van Rhijn L.W., Witlox A.M. Clinical Outcome Following Total Joint Replacement and Arthrodesis for Hallux Rigidus: A Systematic Review. JBJS Rev., 2017, vol. 5, no. 11, p. e2. DOI: 10.2106/JBJS.RVW.17.00032.

22.Chraim M., Bock P., Alrabai H.M., Trnka H.J. Long-term outcome of first metatarsophalangeal joint fusion in the treatment of severe hallux rigidus. Int. Orthop., 2016, vol. 40, no. 11, pp. 2401-2408. DOI: 10.1007/s00264-016-3277-1.

23.Brewster M. Does total joint replacement or arthrodesis of the first metatarsophalangeal joint yield better functional results? A systematic review of the literature. J. Foot Ankle Surg., 2010, vol. 49, no. 6, pp. 546-552. DOI: 10.1053/j.jfas.2010.07.003.

24.Coughlin M.J., Grebing B.R., Jones C.P. Arthrodesis of the first metatarsophalangeal joint for idiopathic hallux valgus: intermediate results. Foot Ankle Int., 2005, vol. 26, no. 10, pp. 783-792. DOI: 10.1177/107110070502601001.

25.Goucher N.R., Coughlin M.J. Hallux metatarsophalangeal joint arthrodesis using dome-shaped reamers and dorsal plate fixation: a prospective study. Foot Ankle Int., 2006, vol. 27, no. 11, pp. 869-876. DOI: 10.1177/107110070602701101.

26.Wanivenhaus F., Espinosa N., Tscholl P.M., Krause F., Wirth S.H. Quality of Early Union After First Metatarsophalangeal Joint Arthrodesis. J. Foot Ankle Surg., 2017, vol. 56, no. 1, pp. 50-53. DOI: 10.1053/j.jfas.2016.09.001.

27.Korim M.T., Mahadevan D., Ghosh A., Mangwani J. Effect of joint pathology, surface preparation and fixation methods on union frequency after first metatarsophalangeal joint arthrodesis: A systematic review of the English literature. Foot Ankle Surg., 2017, vol. 23, no. 3, pp. 189-194. DOI: 10.1016/j.fas.2016.05.317.

28.Brodsky J.W., Passmore R.N., Pollo F.E., Shabat S. Functional outcome of arthrodesis of the first metatarsophalangeal joint using parallel screw fixation. Foot Ankle Int., 2005, vol. 26, no. 2, pp. 140-146. DOI: 10.1177/107110070502600205.

29.Da Cunha R.J., MacMahon A., Jones M.T., Savenkov A., Deland J., Roberts M., Levine D., Elliot A., Kennedy J., Drakos M., Ellis S.J. Return to Sports and Physical Activities After First Metatarsophalangeal Joint Arthrodesis in Young Patients. Foot Ankle Int., 2019, vol. 40, no. 7, pp. 745-752. DOI: 10.1177/1071100719842799.

Received: 24.05.2019

\section{Information about the authors:}

1.Denis V. Ilchenko, M.D.,

European Clinic of Sports Traumatology and Orthopaedics (ECSTO), Moscow, Russian Federation,

Email: dilchenko@emcmos.ru

2. Andrey V. Korolev, M.D., Ph.D., European Clinic of Sports Traumatology and Orthopaedics (ECSTO), Moscow, Russian Federation, Peoples' Friendship University of Russia, Moscow, Russian Federation,

European Medical Center (EMC), Moscow, Russian Federation

3. Andrey A. Kardanov, Ph.D., Professor,

Peoples' Friendship University of Russia, Moscow, Russian Federation,

European Clinic of Sports Traumatology and Orthopaedics (ECSTO), Moscow, Russian Federation, Email: akardanov@emcmos.ru 